\title{
UNIVALENT INTERPOLATION IN BESOV SPACES AND SUPERPOSITION INTO BERGMAN SPACES
}

\author{
STEPHEN M. BUCKLEY AND DRAGAN VUKOTIĆ
}

\begin{abstract}
We characterize the superposition operators from an analytic Besov space or the little Bloch space into a Bergman space in terms of the order and type of the symbol. We also determine when these operators are continuous or bounded. Along the way, we prove new non-centered Trudinger-Moser inequalities and solve the problem of interpolation by univalent functions in analytic Besov spaces.
\end{abstract}

\section{INTRODUCTION}

Characterizing the superposition operators $S_{\varphi}$, defined by $S_{\varphi}(f):=\varphi \circ f$, that take one space, $X$, of analytic functions on the disk to another such space, $Y$, where $X \subset Y$, helps us to compare the growth of functions in those spaces. The natural associated questions are:

For which entire functions $\varphi$ is $S_{\varphi}(X) \subset Y$ ?

When is $S_{\varphi}$ continuous (bounded, Montel compact)?

Similar real variable problems have a long history [AZ], but such questions have only recently been studied in complex function theory; see $[\mathrm{CG}],[\mathrm{Ca}],[\mathrm{BFV}],[\mathrm{AMV}],[\mathrm{X}]$, and $[\mathrm{BV}]$.

Here we continue this line of research by considering the superposition operators from analytic Besov space $B^{p}$ and the little Bloch space $\mathcal{B}_{0}$ to Bergman space $A^{q}$. We will review the definitions of all basic spaces in Section 1.

Our first main result characterizes those operators in terms of $E(t)$, the class of entire functions of order less than a positive number $t$, or of order $t$ and finite type.

Theorem 1. Suppose $1<p<\infty$ and $0<q<\infty$. Then $S_{\varphi}\left(B^{p}\right) \subset A^{q}$ if and only if $\varphi \in E(p /(p-1))$, and $S_{\varphi}\left(\mathcal{B}_{0}\right) \subset A^{q}$ if and only if $\varphi \in E(1)$. All superposition operators from $B^{p}$ or $\mathcal{B}_{0}$ to $A^{q}$ are continuous (as maps between metric spaces).

Since $S_{\varphi}$ is nonlinear if $\varphi(z) \not \equiv c z$, there is no reason that continuity and boundedness should be equivalent. Indeed there are continuous unbounded superposition operators

Date: 28 September, 2006.

2000 Mathematics Subject Classification. 47H30, 31C25, 30H05.

This research was supported by MCyT grant BFM2003-07294-C02-01, Spain. The first author was also supported by Enterprise Ireland and Science Foundation Ireland. 
from $B^{p}$ to $A^{q}$, as revealed by the following characterization of boundedness and compactness. Below, $E_{0}(t)$ is the class of entire functions of order less than $t$, or of order $t$ and type zero.

Theorem 2. Suppose $1<p<\infty$ and $0<q<\infty$. Then $S_{\varphi}$ is bounded from $B^{p}$ to $A^{q}$ if and only if $\varphi \in E_{0}(p /(p-1))$, and $S_{\varphi}$ is bounded from $\mathcal{B}_{0}$ to $A^{q}$ if and only if $\varphi \in E_{0}(1)$. All such bounded operators are Montel compact.

Theorems 1 and 2 are proved in Section 4, after some preparatory work. We note that these results do not seem to follow in any obvious way from the known descriptions of interpolation sequences such as the unpublished manuscript by Donald Marshall and Carl Sundberg, or the paper of Böe [Boe].

The key to proving continuity in Theorem 1 is the following non-centered TrudingerMoser inequality which may be of independent interest; see Section 2.

THEOREM 3. Let $1<p<\infty$ and, for $\alpha, r>0$ and $f \in B^{p}$, define

$$
M_{\alpha ; r, f}:=\sup \left\{\int_{\mathbb{D}} \exp \left(\alpha|g|^{p /(p-1)}\right) d A:\|g-f\|_{B^{p}} \leq r\right\} .
$$

Also let $M_{\alpha}:=M_{\alpha ; 1,0}$ and $s_{p}:=\sup \left\{\alpha: M_{\alpha}<\infty\right\}$.

(a) $0<s_{p}<\infty$. Moreover, there exists $c=c(p)>0$ such that $E_{c} \leq 2$.

(b) Let $\alpha>0$ and $f \in B^{p}$. Then $M_{\alpha ; r, f}<\infty$ for all $0 \leq r<r_{0}:=\left(s_{p} / \alpha\right)^{(p-1) / p}$, and $M_{\alpha ; r, f}=\infty$ for all $r>r_{0}$.

The main novelty in the above theorem is its non-centered nature; note that the conclusions are independent of the "center point" $f$. Trudinger-Moser inequalities, which give some type of exponential integrability of a function whose derivative satisfies some integrability condition, go back to Beurling's thesis $[\mathrm{Be}]$; see also $[\mathrm{CM}]$, $[\mathrm{Ch}$, Lecture 3], and [BO]. Also in Section 2, we obtain a sharper variant of Theorem 3 for the Dirichlet space $\mathcal{D}$ and also a variant for $\mathcal{B}_{0}$.

The key to proving boundedness in Theorem 2 is the following result on growth rate of $B^{p}$ functions; see Section 3.

THEOREM 4. Given a number $p \in(1, \infty)$ and a decreasing positive sequence $\left\{r_{n}\right\} \in l^{p}$, with $r_{1} \leq 1$, there exists a univalent function $f \in B^{p}$ and a constant $c=c(p)>0$ such that $f(0)=0$ and

$$
\left|f\left(z_{n}\right)\right|^{p /(p-1)} \geq c r_{n} \log \frac{1}{1-\left|z_{n}\right|}, \quad n \in \mathbb{N},
$$

for some sequence of points $\left\{z_{n}\right\}$ in the disk such that $\left|z_{n}\right| \rightarrow 1$.

Moreover, (1) can be obtained with the additional requirement that $f\left(z_{n}\right)=w_{n}$ for any prescribed sequence $\left\{w_{n}\right\}_{n=1}^{\infty}$ in the plane with the following properties for all $n \in \mathbb{N}$ :

(i) $w_{0}=0,\left|w_{1}\right|>1$;

(ii) $2\left|w_{n}\right|<\left|w_{n+1}\right|$;

(iii) $0 \leq \arg w_{n}<\pi / 4$. 
The fact that the maps constructed in the above theorem interpolate the prescribed fast-growing values $w_{n}$ at the same points $z_{n}$ is a novel aspect of this theorem, and essential to the proof of Theorem 2. The theorem proves sharpness in a strong sense of the well-known estimate of Holland and Walsh [HW] on the order of growth of a function in $B^{p}, p>1$ :

$$
|f(z)|=o\left(\left(\log \frac{1}{1-|z|}\right)^{1-1 / p}\right), \quad \text { as } \quad|z| \rightarrow 1^{-} .
$$

Also in Section 3, a $\mathcal{B}_{0}$ variant of Theorem 4 is proved.

\section{BACKGROUND}

Throughout, $\mathbb{C}$ will denote the complex plane, $\mathbb{D}$ the unit disk $\{z \in \mathbb{C}:|z|<1\}, \mathbb{T}$ the unit circle $\{z \in \mathbb{C}:|z|=1\}$, and $d A(z):=\pi^{-1} d x d y=\pi^{-1} r d r d \theta$ the normalized area measure on $\mathbb{D}$. A disk of radius $r$ centered at $w$ is denoted $D(w, r)$, while $[z, w]$ means the line segment from the point $z$ to the point $w$.

Given two positive expressions $A, B$, we write $A \lesssim B$, or $B \gtrsim A$, to mean that $A \leq C B$ for some constant $C$ dependent only on allowed parameters (which should be clear from the context). We write $A \approx B$ to mean that $A \lesssim B \lesssim A$. We write $C=C(p, q, \ldots)$ to indicate that $C$ depends only on the parameters $p, q, \ldots$

We say that a complex function $\varphi$ acts by superposition from one class of analytic functions $X$ to another class $Y$ if $\varphi \circ f \in Y$ whenever $f \in X$; note that $\varphi$ must be entire if $X$ contains the linear functions. In this case we define the superposition operator $S_{\varphi}: X \rightarrow Y$ with symbol $\varphi$ by $S_{\varphi}(f):=\varphi \circ f$. If $X$ and $Y$ have associated metrics, we define continuity of $S_{\varphi}$ in the usual sense, and we say that $S_{\varphi}$ is bounded if it maps bounded sets into bounded sets and Montel compact if it maps bounded sets into relatively compact sets.

1.1. Hyperbolic metric. The hyperbolic distance between the points $z$ and $w$ in $\mathbb{D}$ is defined as

$$
\varrho(z, w):=\inf _{\gamma} \int_{\gamma} \frac{|d \zeta|}{1-|\zeta|^{2}}=\frac{1}{2} \log \frac{1+\left|\frac{z-w}{1-\bar{z} w}\right|}{1-\left|\frac{z-w}{1-\bar{z} w}\right|},
$$

where the infimum is taken over all rectifiable curves $\gamma$ in $\mathbb{D}$ joining $z$ and $w$. The hyperbolic metric $\varrho_{\Omega}$ on a simply connected domain $\Omega \subsetneq \mathbb{C}$ is now defined by pullback: given a univalent mapping $f$ of $\mathbb{D}$ onto $\Omega$, we have

$$
\rho_{\Omega}(f(z), f(w)):=\rho(z, w)=\inf _{\Gamma} \int_{f^{-1}(\Gamma)} \frac{|d \zeta|}{1-|\zeta|^{2}},
$$

where the infimum is taken over all rectifiable curves $\Gamma$ in $\Omega$ from $f(z)$ to $f(w)$. This definition is easily seen to be independent of $f$. It follows that if $f(0)=0$ then

$$
\varrho_{\Omega}(0, f(z))=\varrho(0, z) \geq \frac{1}{2} \log \frac{1}{1-|z|}, \quad \text { for all } z \in \mathbb{D} .
$$


On the other hand, it is well-known that

$$
\varrho_{\Omega}\left(w_{1}, w_{2}\right) \leq \inf _{\Gamma} \int_{\Gamma} \frac{|d w|}{\operatorname{dist}(w, \partial \Omega)},
$$

where the infimum is taken over all rectifiable curves $\Gamma$ in $\Omega$ from $w_{1}$ to $w_{2}$. We refer the reader to $[\mathrm{P}$, Chapter 4] for all these properties.

1.2. Hardy and Bergman spaces. We denote by $H^{p}$ the well-known Hardy space of functions analytic in $\mathbb{D}$ for which

$$
\|f\|_{H^{p}}^{p}:=\sup _{0<r<1}\left(\int_{0}^{2 \pi}\left|f\left(r e^{i \theta}\right)\right|^{p} \frac{d \theta}{2 \pi}\right)^{1 / p}<\infty .
$$

All $H^{p}$ functions have radial limits, written as $f\left(e^{i \theta}\right)$, almost everywhere on $\mathbb{T}$.

The Bergman space $A^{p}, 0<p<\infty$, is the space of functions analytic on $\mathbb{D}$ with

$$
\|f\|_{A^{p}}^{p}:=\int_{\mathbb{D}}|f(z)|^{p} d A(z)<\infty .
$$

For more on Bergman spaces, see [HKZ] and [DS]. We only mention a few facts about them that we need.

The space $A^{p}$ is a Banach space when $p \geq 1$, and a complete metric space with metric $d_{p}(f, g):=\|f-g\|_{A^{p}}^{p}$ when $p<1$. For all $0<q<p<\infty, A^{p}$ is compactly embedded in $A^{q}$; see $[\mathrm{Ax}]$. Using subharmonicity, it is readily seen that $\|f\|_{A^{p}} \leq\|f\|_{H^{p}}$ for all $f \in H^{p}$, and that

$$
(1-|z|)^{2}|f(z)|^{p} \leq\|f\|_{A^{p}}^{p}, \quad f \in A^{p}, z \in \mathbb{D} .
$$

Defining the weighted Dirichlet-type space $D_{p}^{p}$ as the set of all analytic functions in $\mathbb{D}$ with finite norm

$$
\|f\|_{D_{p}^{p}}^{p}:=|f(0)|^{p}+(p+1) \int_{\mathbb{D}}\left|f^{\prime}(z)\right|^{p}\left(1-|z|^{2}\right)^{p} d A(z)
$$

a theorem of Hardy and Littlewood [HL] essentially shows that $D_{p}^{p}$ and $A^{p}$ coincide and have comparable norms for all $0<p<\infty$; for a proof, see [D, Theorem 5.6] and the method of [D, Theorem 5.5].

1.3. Besov and Bloch spaces. The (analytic) Besov space $B^{p}, 1<p<\infty$, is the Banach space of functions analytic on $\mathbb{D}$ for which

$$
\|f\|_{B^{p}}:=|f(0)|+\left((p-1) \int_{\mathbb{D}}\left|f^{\prime}(z)\right|^{p}\left(1-|z|^{2}\right)^{p-2} d A(z)\right)^{1 / p}<\infty .
$$

The Bloch space $\mathcal{B}$ is the Banach space of all functions analytic in $\mathbb{D}$ for which

$$
\|f\|_{\mathcal{B}}:=|f(0)|+\sup _{z \in \mathbb{D}}\left(1-|z|^{2}\right)\left|f^{\prime}(z)\right|<\infty .
$$

The little Bloch space $\mathcal{B}_{0}$ is the set of all $f \in \mathcal{B}$ such that $\lim _{|z| \rightarrow 1^{-}}\left(1-|z|^{2}\right)\left|f^{\prime}(z)\right|=0$. It is the natural limit as $p \rightarrow \infty$ of $B^{p}$, as well as the closure of the polynomials in $\mathcal{B}$. 
For more on these spaces and their operators, see [AFP], [HW], [Z], [BFV], [DGV], and [Boe]. Here we mention only a few facts that we need.

First we note that $B^{p} \subset B^{q}$ whenever $p<q$, and that these spaces are conformally invariant: if $f \in B^{p}$, then $f \circ \phi \in B^{p}$ for every disk automorphism $\phi$. The Dirichlet space is $\mathcal{D}:=B^{2}$. We note that $\mathcal{D} \subset H^{p}, 0<p<\infty$; see [D, Chapter 6 , Exercise 7], for instance.

By integrating the inequality $\left|f^{\prime}(z)\right| \leq\|f\|_{\mathcal{B}} /\left(1-|z|^{2}\right)$ along a line segment, we get

$$
|f(z)-f(0)| \leq|f(0)|+\left(\|f\|_{\mathcal{B}}-|f(0)|\right) \varrho(0, z), \quad z \in \mathbb{D} .
$$

Given a simply connected domain $\Omega \subsetneq \mathbb{C}$, we denote by $d_{\Omega}(w)$ the distance from $w$ to $\partial \Omega$. The following Koebe distortion estimates for a univalent map $f: \mathbb{D} \rightarrow \Omega$ are well known [P, Corollary 1.4]:

$$
d_{\Omega}(f(z)) \leq\left|f^{\prime}(z)\right|\left(1-|z|^{2}\right) \leq 4 d_{\Omega}(f(z)),
$$

and they readily imply the following lemma; for proofs of different parts, see $[\mathrm{ACP}]$, $[\mathrm{W}],[\mathrm{BFV}]$, and $[\mathrm{DGV}]$.

Lemma A. Let $f$ be a univalent map of $\mathbb{D}$ onto a simply connected domain $\Omega$. Then

(a) $f \in B^{p}, 1<p<\infty$, if and only if $\int_{\Omega} d_{\Omega}(w)^{p-2} d A(w)<\infty$;

(b) $f \in \mathcal{B}$ if and only if $\sup _{w \in \Omega} d_{\Omega}(w)<\infty$;

(c) $f \in \mathcal{B}_{0}$ if and only if $\lim _{\left|f^{-1}(w)\right| \rightarrow 1, w \in \Omega} d_{\Omega}(w)=0$.

We shall need the test functions

$$
f_{r, p}:=c_{r, p} \log (1 /(1-r z))\left(\log \left(1 /\left(1-r^{2}\right)\right)^{-1 / p}, \quad 0<r<1,1<p<\infty,\right.
$$

where $c_{r, p}>0$ is chosen so that $\left\|f_{r, p}\right\|_{B^{p}}=1$. Denoting by $\Gamma$ the classical gamma function, we note that $c_{r, p}^{p}$ is asymptotic to $(\Gamma(p / 2))^{2} / \Gamma(p-1)$ as $r \rightarrow 1^{-}$. This can be seen by checking the proof of Theorem 1.7 in [HKZ].

\section{Inequalities of TRUdinger-Moser TYPE}

In this section, we prove non-centered variants of existing Trudinger-Moser type inequalities. We begin with the proof of Theorem 3 .

Proof of Theorem 3. The fact that there exists $c>0$ such that $E_{c} \leq 2$ is a restatement of the case $\alpha=0$ of [BFV, Theorem 23]. Thus $s_{p}>0$, and we need only show that $s_{p}<\infty$. Consider the functions $f_{r, p}$ defined by (8), and take $r=1-\varepsilon$ for $0<\varepsilon<1 / 2$. The asymptotic formula for $c_{r, p}$ tells us that there is a number $b=b(p)>0$ such that $f_{1-\varepsilon, p}(z) \geq b \log ^{1-1 / p}(1 / \varepsilon)$ in the disk $D(r, \varepsilon)$. It follows that if $\alpha$ is sufficiently large, then $\exp \left(\alpha\left|f_{1-\varepsilon, p}\right|^{p /(p-1)}\right) \geq \varepsilon^{-3}$ on $D(r, \varepsilon)$, and so

$$
\int_{D} \exp \left(\alpha\left|f_{1-\varepsilon, p}\right|^{p /(p-1)}\right) d A \rightarrow \infty \quad \text { as } \varepsilon \rightarrow 0 .
$$

Letting $\varepsilon \rightarrow 0$, we see that $M_{\alpha}=\infty$, and so $s_{p}<\infty$ as required. 
We now prove (b). Suppose that $\|f-g\|_{B^{p}} \leq r<r_{0}$, and write $r_{1}=\left(r+r_{0}\right) / 2$, so that $r<r_{1}<r_{0}$. Since the polynomials are dense in $B^{p}$ [AFP, Proposition 2], we can find a polynomial $h$ so that $\|f-h\|_{B^{p}} \leq r_{1}-r$. Next note the following elementary inequality for real numbers, which can be proved by calculus:

$$
(a+b)^{s} \leq C_{\varepsilon} a^{s}+c_{\varepsilon} b^{s}, \quad a, b, \varepsilon>0, s>1,
$$

where $C_{\varepsilon}=(1+1 / \varepsilon)^{s-1}$ and $c_{\varepsilon}=(1+\varepsilon)^{s}$. Applying this inequality with $a=|h(z)|$, $b=|g(z)-h(z)|$, and $s=p /(p-1)$, we see that

$$
\int_{\mathbb{D}} \exp \left(\alpha|g|^{p /(p-1)}\right) d A \leq \int_{\mathbb{D}} \exp \left(\alpha C_{\varepsilon}|h|^{p /(p-1)}\right) \cdot \exp \left(\alpha c_{\varepsilon}|g-h|^{p /(p-1)}\right) d A
$$

Since $h$ is a polynomial, the first factor in this integrand is bounded and can be discarded. To bound the resulting integral, we choose $\varepsilon>0$ so small that $t_{1} \leq$ $\left(s_{p} / \alpha c_{\varepsilon}\right)^{(p-1) / p}$. Now $\|g-h\|_{B^{p}} \leq t_{1}$, and so $\left\|(g-h) / t_{1}\right\|_{B^{p}} \leq 1$. Applying part (a) to $(g-h) / t_{1}$, and noting that $\alpha c_{\varepsilon} t_{1}^{p /(p-1)} \leq \alpha$, we see that

$$
\int_{\mathbb{D}} \exp \left(\alpha c_{\varepsilon}|g-h|^{p /(p-1)}\right) d A \leq M_{\alpha}<\infty
$$

Thus $M_{\alpha ; r, f}<\infty$, as required.

Suppose instead that $r>r_{0}$. We must prove that $M_{\alpha ; r, f}=\infty$. Let $r_{1}:=\left(r+r_{0}\right) / 2$ and $\tau:=\left(r_{1} / r_{0}\right)^{p /(p-1)}>1$. Pick a polynomial $h$ so close to $f$ that $\|f-h\| \leq r-r_{1}$. By definition of $s_{p}$, we can choose functions $\left\{u_{n}\right\}$ in the unit ball of $B^{p}$ such that

$$
\int_{\mathbb{D}} \exp \left(\tau^{1 / 2} s_{p}\left|u_{n}\right|^{p /(p-1)}\right) d A \rightarrow \infty \quad \text { as } n \in \mathbb{N} .
$$

Letting $v_{n}:=r_{1} u_{n}=\left(\tau s_{p} / \alpha\right)^{(p-1) / p} u_{n}$, this last inequality can be rewritten as

$$
\int_{\mathbb{D}} \exp \left(\tau^{-1 / 2} \alpha\left|v_{n}\right|^{p /(p-1)}\right) d A \rightarrow \infty \quad \text { as } n \in \mathbb{N} \text {. }
$$

We now define $g_{n}:=v_{n}+h$. Since $\left|v_{n}\right| \leq|h|+\left|g_{n}\right|$, we can apply (9) to get that

$$
\exp \left(\tau^{-1 / 2} \alpha\left|v_{n}\right|^{p /(p-1)}\right) \leq \exp \left(C_{\varepsilon} \tau^{-1 / 2} \alpha|h|^{p /(p-1)}\right) \cdot \exp \left(c_{\varepsilon} \tau^{-1 / 2} \alpha\left|g_{n}\right|^{p /(p-1)}\right) .
$$

Now $h$ is a polynomial, so the first factor on the right is bounded. Thus it follows from (10) that for every $\varepsilon>0$,

$$
\int_{\mathbb{D}} \exp \left(c_{\varepsilon} \tau^{-1 / 2} \alpha\left|g_{n}\right|^{p /(p-1)}\right) d A \rightarrow \infty \quad \text { as } n \in \mathbb{N} .
$$

By choosing $\varepsilon=\tau^{(p-1) / 2 p}-1$, we deduce that

$$
\int_{\mathbb{D}} \exp \left(\alpha\left|g_{n}\right|^{p /(p-1)}\right) d A \rightarrow \infty \quad \text { as } n \in \mathbb{N}
$$

But

$$
\left\|g_{n}-f\right\|_{B^{p}}=\left\|v_{n}+(h-f)\right\|_{B^{p}} \leq\left\|v_{n}\right\|_{B^{p}}+\|h-f\|_{B^{p}} \leq r_{1}+\left(r-r_{1}\right)=r,
$$


and so we conclude that $M_{\alpha ; r, f}=\infty$.

Let us make a couple of remarks on the above theorem. First note that by expanding the exponential function as a power series, it follows from (a) that for every $1<p<\infty$ and $0<q<\infty$, there exists $C=C(p, q)$ such that

$$
\|f\|_{A_{q}} \leq C\|f\|_{B^{p}}, \quad f \in B^{p} .
$$

Although Theorem 3(b) is a sharp non-centered variant of [BFV, Theorem 23], it involves the unknown quantity $s_{p}$ whose value we do not in general know. An exception is that $s_{2}=1$, as follows readily from the famous Chang-Marshall inequality [CM, Theorem 1]. We now state a non-centered variant of that result.

TheOREM 5. For $\alpha, r>0$ and $f \in \mathcal{D}$, let

$$
\mathcal{M}_{\alpha ; r, f}:=\sup \left\{\int_{\mathbb{T}} \exp \left(\alpha\left|g\left(e^{i \theta}\right)\right|^{2}\right) d \theta:\|g-f\|_{\mathcal{D}} \leq r\right\} .
$$

Also let $\mathcal{M}_{\alpha}:=\mathcal{M}_{\alpha ; 1,0}$ and $s_{2}:=\sup \left\{\alpha: \mathcal{M}_{\alpha}<\infty\right\}$.

(a) $\mathcal{M}_{\alpha}<\infty$ if and only if $\alpha \leq 1$; in particular $s_{2}=1$.

(b) Let $\alpha>0$ and $f \in \mathcal{D}$. Then $\mathcal{M}_{\alpha ; r, f}<\infty$ for all $0 \leq r<r_{0}:=\alpha^{-1 / 2}$, and $\mathcal{M}_{\alpha ; r, f}=\infty$ for all $r>r_{0}$.

Proof. Let us write $I_{f}:=\int_{\mathbb{T}} \exp \left(\left|f\left(e^{i \theta}\right)\right|^{2}\right) d \theta, f \in \mathcal{D}$. Part (a) is a slight improvement on the Chang-Marshall inequality, which tells us that there exists a constant $K<\infty$ such that $I_{f} \leq K$ whenever $f \in \mathcal{D},\|f\|_{\mathcal{D}} \leq 1$, and $f(0)=0$ [CM, Theorem 1].

We deduce a uniform bound for $I_{f}$ over all $f \in \mathcal{D},\|f\|_{\mathcal{D}} \leq 1$. Such a bound is trivial when $f$ is constant, so we assume that $f$ is nonconstant and write $g:=f-f(0)$. Then $|f(0)|<1$ and

$$
\int_{\mathbb{T}} \exp \left(\left|f\left(e^{i \theta}\right)\right|^{2}\right) d \theta \leq e \int_{\mathbb{T}} \exp \left(\left|g\left(e^{i \theta}\right)\right|^{2}\right) \cdot \exp \left(2|f(0)|\left|g\left(e^{i \theta}\right)\right|\right) d \theta .
$$

Writing $\beta:=1 /(1-|f(0)|)$, we note that $\|\beta g\|_{\mathcal{D}} \leq 1$ and $1+|f(0)| \leq \beta^{2}$. Therefore

$$
\int_{\mathbb{T}} \exp \left(\left|g\left(e^{i \theta}\right)\right|^{2}\right) \cdot \exp \left(|f(0)|\left|g\left(e^{i \theta}\right)\right|^{2}\right) d \theta \leq \int_{\mathbb{T}} \exp \left(\beta^{-2}\left|g\left(e^{i \theta}\right)\right|^{2}\right) \leq K .
$$

Thus if make separate estimates for those points $\theta \in \mathbb{T}$ where $\left|g\left(e^{i \theta}\right)\right|$ is less than or equal to, or greater than, 2 , it follows from (12) that

$$
\int_{\mathbb{T}} \exp \left(\left|f\left(e^{i \theta}\right)\right|^{2}\right) d \theta \leq e^{9}+K e .
$$

We have shown that $E_{1} \leq e^{9}+K e$.

The fact that $\mathcal{M}_{\alpha}=\infty$ when $\alpha>1$ is well-known, and goes back to the work of Beurling [Be]. In fact he proved this using the functions $f_{r, 2}$ defined in (8).

The proof of (b) is very similar to the proof of Theorem 3(b), so we omit it.

Finally in this section, we state a little Bloch analogue of Theorem 3. 
Theorem 6. For $\alpha, r>0$ and $f \in \mathcal{B}_{0}$, define

$$
m_{\alpha ; r, f}:=\sup \left\{\int_{\mathbb{D}} \exp (\alpha|g|) d A:\|g-f\|_{\mathcal{B}_{0}} \leq r\right\} .
$$

Also let $m_{\alpha}:=m_{\alpha ; 1,0}$ and $s_{\infty}:=\sup \left\{\alpha: m_{\alpha}<\infty\right\}$.

(a) $2 \leq s_{\infty} \leq 4$.

(b) Let $\alpha>0$ and $f \in \mathcal{B}_{0}$. Then $m_{\alpha ; r, f}<\infty$ for all $0 \leq r<r_{0}:=s_{\infty} / \alpha$, and $m_{\alpha ; r, f}=\infty$ for all $r>r_{0}$.

Proof. The functions $f_{r}(z):=\frac{1}{2} \log ((1+r z) /(1-r z)), 0<r<1$, all lie in the unit ball of $\mathcal{B}_{0}$. By estimating $f_{r}$ on the disk $D(r, 1-r)$ and letting $r \rightarrow 1^{-}$, it is easy to see that $m_{\alpha}=\infty$ for $\alpha>4$. The estimate $s_{\infty} \geq 2$ easily follows from (7).

For part (b), we use the fact that polynomials are dense in $\mathcal{B}_{0}$ and argue as in Theorem 3(b), except that we do not need (9).

\section{UNIVALENT INTERPOLATION AT A PRESCRIBED GROWTH RATE}

We construct a family of examples of functions in $B^{p}$ which prove that the HollandWalsh estimate (2) is quite sharp. These examples achieve a prescribed "little-oh" rate of growth on an infinite sequence of points and, on that same sequence, take on prescribed values which grow at a fast exponential rate. This last feature will be fundamental in Section 4.

Proof of Theorem 4. By (ii), we have $\left|w_{n}\right| \rightarrow \infty$ and $\left|w_{n+1}-w_{n}\right| \rightarrow \infty$ as $n \rightarrow \infty$. Also by (ii), we readily see that there exists $C=C(p)$ such that

$$
\sum_{k=1}^{n} r_{k}^{-1}\left|w_{k}-w_{k-1}\right|^{p /(p-1)} \leq C r_{n}^{-1}\left|w_{n}\right|^{p /(p-1)}, \quad n \in \mathbb{N} .
$$

Writing $h_{n}:=r_{n}\left|w_{n}-w_{n-1}\right|^{-1 /(p-1)}, n \in \mathbb{N}$, we have $h_{n}<r_{n}, n \in \mathbb{N}$. Now define the domain $\Omega:=\bigcup_{n=0}^{\infty}\left(D\left(w_{n}, r_{n}\right) \cup R_{n+1}\right)$, where

$$
R_{n}:=\bigcup_{w \in\left[w_{n-1}, w_{n}\right]} D\left(w, h_{n}\right), \quad n \in \mathbb{N},
$$

By construction, $R_{n}$ and $R_{m}$ intersect if and only if $|m-n| \leq 1$. It is also straightforward to show that $\Omega$ is simply connected, since it is the union of an ascending chain of simply connected domains; see also Step 3 of the proof of [DGV, Theorem 2.1].

Let $f$ be a conformal map of $\mathbb{D}$ onto $\Omega$ that fixes the origin. By our assumptions on $r_{n}$ and our choice of $h_{n}$, it is clear that

$$
\sum_{n=1}^{\infty} r_{n}^{p}+\sum_{n=1}^{\infty} h_{n}^{p-1}\left|w_{n}-w_{n-1}\right|<\infty
$$


But using Lemma A, it is not hard to see that this last inequality is exactly what we need to conclude that $f \in B^{p}$; for more details, see a similar construction in [BFV, Proposition 7].

Writing $z_{n}:=f^{-1}\left(w_{n}\right)$, it is clear that $\left|z_{n}\right| \rightarrow 1^{-}$as $n \rightarrow \infty$. Using inequality (3), the triangle inequality, property (4), the definitions of $\Omega$ and $h_{n}$, and estimate (13), we obtain

$$
\begin{aligned}
\log \frac{1}{1-\left|z_{n}\right|} & \lesssim \varrho_{\Omega}\left(0, w_{n}\right) \leq \sum_{k=1}^{n} \varrho_{\Omega}\left(w_{k-1}, w_{k}\right) \\
& \lesssim \sum_{k=1}^{n} \frac{\left|w_{k}-w_{k-1}\right|}{h_{k}}=\sum_{k=1}^{n} r_{k}^{-1}\left|w_{k}-w_{k-1}\right|^{p /(p-1)} \lesssim r_{n}^{-1}\left|w_{n}\right|^{p /(p-1)}
\end{aligned}
$$

This proves (1), and we are done.

We now state a $\mathcal{B}_{0}$ variant of this last theorem; compare with the result for $\mathcal{B}$ in [AMV, Lemma 2].

LEMMA 7. Given a decreasing positive sequence $\left\{r_{n}\right\}$, with $r_{1} \leq 1$, there exists a univalent function $f \in \mathcal{B}_{0}$ and a constant $c>0$ such that $f(0)=0$ and

$$
\left|f\left(z_{n}\right)\right| \geq c r_{n} \log \frac{1}{1-\left|z_{n}\right|}, \quad n \in \mathbb{N}
$$

for some sequence of points $\left\{z_{n}\right\}$ in the disk such that $\left|z_{n}\right| \rightarrow 1$.

Moreover, (14) can be obtained with the additional requirement that $f\left(z_{n}\right)=w_{n}$ for any prescribed sequence $\left\{w_{n}\right\}_{n=1}^{\infty}$ in the plane with the following properties for all $n \in \mathbb{N}$ :

(i) $w_{0}=0,\left|w_{1}\right|>1$;

(ii) $2\left|w_{n}\right|<\left|w_{n+1}\right|$;

(iii) $0 \leq \arg w_{n}<\pi / 4$.

Proof. The argument is similar to, but easier than, Theorem 4, so we shall be sketchy. Let $\Omega$ be the simply connected domain $\bigcup_{n=1}^{\infty} R_{n}$, where

$$
R_{n}:=\bigcup_{w \in\left[w_{n-1}, w_{n}\right]} D\left(w, r_{n}\right), \quad n \in \mathbb{N}
$$

Let $f$ be a conformal map of $\mathbb{D}$ onto $\Omega$ that fixes the origin, so that $f \in \mathcal{B}_{0}$ according to Lemma A.

Writing $z_{n}:=f^{-1}\left(w_{n}\right)$, it is clear that $\left|z_{n}\right| \rightarrow 1^{-}$as $n \rightarrow \infty$. As before, we see that

$$
\log \frac{1}{1-\left|z_{n}\right|} \lesssim \varrho_{\Omega}\left(0, w_{n}\right) \leq \sum_{k=1}^{n} \varrho_{\Omega}\left(w_{k-1}, w_{k}\right) \lesssim \sum_{k=1}^{n} r_{k}^{-1}\left|w_{k}-w_{k-1}\right| \lesssim r_{n}^{-1}\left|w_{n}\right|
$$

This proves (14), and we are done. 


\section{Superposition operators from Besov spaces to Bergman spaces}

If $S_{\varphi}\left(A^{q}\right)$ is a subset of $B^{p}$, or of $\mathcal{B}_{0}$, then $S_{\varphi}\left(A^{q}\right) \subset \mathcal{B}$ and so, according to [AMV], the function $\varphi$ must be identically zero. In this section, we tackle the reverse action. More precisely, we characterize the superposition operators $S_{\varphi}$ that act, or are bounded, or are continuous, from $B^{p}$ to $A^{q}$ in terms of the order and type of the symbol $\varphi$.

Since an $A^{q}$ function grows as the power $-1 / q$ of the distance to the boundary and a $B^{p}$ function grows at most like the power $1-1 / p$ of the logarithm of the reciprocal value of the distance, and both estimates are sharp, it is to be expected that $B^{p}$ should be "contained exponentially" in $A^{q}$. This is indeed the case.

Recall that the order of a non-constant entire function $\varphi$ is

$$
\rho:=\limsup _{r \rightarrow \infty} \frac{\log \log M(r, \varphi)}{\log r},
$$

where $M(r, \varphi):=\max \{|\varphi(z)|:|z|=r\}$. The type $\sigma$ of an entire function $\varphi$ of order $\rho$ $(0<\rho<\infty)$ is

$$
\sigma:=\limsup _{r \rightarrow \infty} \frac{\log M(r, \varphi)}{r^{\rho}}
$$

See the first two chapters of [Boa] for more on entire functions.

Recall that $E(t)$ consists of all entire functions of order less than $t$, or of order $t$ and finite type. Equivalently, $\varphi \in E(t)$ if and only if there exists a constant $\alpha$ such that $|\varphi(z)| \leq \exp \left(\alpha|z|^{t}\right)$, for all sufficiently large $z \in \mathbb{C}$. Also recall that $E_{0}(t)$ consists of all entire functions of order less than $t$, or order $t$ and type 0 , that is $\varphi \in E_{0}(t)$ if and only if for all $\varepsilon>0$, there exists $R$ such that $|\varphi(z)| \leq \exp \left(\varepsilon|z|^{t}\right)$, for all $|z| \geq R$.

4.1. Operators acting (continuously) from $B^{p}$ to $A^{q}$. Here we prove Theorem 1 , thereby characterizing the (continuous) superposition operators from $B^{p}$ to $A^{q}$. Let us first note that a part of this theorem follows from [BFV, Theorem 26]. Indeed if we take $\beta=q$ in that result, and note that $D_{q}^{q}=A^{q}$, then for the case $0<q \leq p$, we get that $S_{\varphi}\left(B^{p}\right) \subset A^{q}$ if and only if $\varphi \in E(p /(p-1))$. However, the case $q>p$ is not covered by that result, and continuity of such operators was not addressed either in $[\mathrm{BFV}]$. The superposition operators from the Bloch space $\mathcal{B}$ to $A^{q}$ were characterized in [AMV, Theorem 3].

Proof of Theorem 1. Given $\varphi \in E(p /(p-1))$, there exist constants $C, \alpha$ such that

$$
|\varphi(w)| \leq C \exp \left(\alpha|w|^{p /(p-1)}\right), \quad w \in \mathbb{C} .
$$

By the case $r=0$ of Theorem 3(b),

$$
\int_{\mathbb{D}} \mid \varphi\left(\left.f(z)\right|^{q} d A(z) \leq C^{q} \int_{\mathbb{D}} \exp \left(q \alpha|f(z)|^{p /(p-1)}\right) d A(z)<\infty,\right.
$$

whenever $f \in B^{p}$. Thus $S_{\varphi}\left(B^{p}\right) \subset A^{q}$. 
If $f \in \mathcal{B}_{0}$ then similarly the case $r=0$ of Theorem 6 (c) implies that $S_{\varphi}\left(\mathcal{B}_{0}\right) \subset A^{q}$ for all $\varphi \in E(1)$.

We now tackle necessity. In order to arrive at a contradiction, suppose that $S_{\varphi}\left(B^{p}\right) \subset$ $A^{q}$, but that $\varphi \notin E(p /(p-1))$. Without loss of generality we may add a constant to $\varphi$ so that $\varphi(0)=0$, as this will not change the order and type. It follows from (15) that there exists a sequence of points $\left\{w_{n}\right\}_{n=1}^{\infty}$ in the plane such that

$$
\left|\varphi\left(w_{n}\right)\right| \geq \exp \left(n^{2}\left|w_{n}\right|^{p /(p-1)}\right), \quad n \in \mathbb{N} .
$$

Clearly, $\left|w_{n}\right| \rightarrow \infty$ as $n \rightarrow \infty$, so we may extract a convergent subsequence entirely located in an angular sector of aperture $\pi / 4$, rotate it if necessary, and select a further convergent subsequence to obtain a new sequence, labelled again $\left\{w_{n}\right\}$ so as to satisfy the assumptions of Theorem 4, after adding the point $w_{0}=0$ to the sequence. We choose

$$
r_{n}:=\frac{1}{n} \quad \text { and } \quad h_{n}:=\frac{1}{n\left|w_{n+1}-w_{n}\right|^{1 /(p-1)}} .
$$

Let again $f$ be a univalent map of $\mathbb{D}$ onto the corresponding domain $\Omega$ and let $z_{n}:=$ $f^{-1}\left(w_{n}\right)$, with $z_{0}=0$. Our choice of $r_{n}$ and $h_{n}$ above readily yield that $f \in B^{p}$. By (5), (17), and (1), we have

$$
\begin{aligned}
\|\varphi \circ f\|_{A^{q}}^{q} & \geq\left(1-\left|z_{n}\right|\right)^{2}\left|\varphi\left(f\left(z_{n}\right)\right)\right|^{q} \\
& \geq\left(1-\left|z_{n}\right|\right)^{2} \exp \left(q n^{2}\left|f\left(z_{n}\right)\right|^{p /(p-1)}\right) \\
& \geq\left(1-\left|z_{n}\right|\right)^{2-c q n} \\
& \geq\left(1-\left|z_{n}\right|\right)^{-1}
\end{aligned}
$$

whenever $n>3 / c q$. This last expression is unbounded as $n \rightarrow \infty$, giving the desired contradiction.

In the case of the little Bloch space, suppose for the sake of contradiction that $S_{\varphi}\left(\mathcal{B}_{0}\right) \subset A^{q}$, but that $\varphi \notin E(1)$. Then there is a sequence of points $\left\{w_{n}\right\}_{n=1}^{\infty},\left|w_{n}\right| \rightarrow \infty$, such that

$$
\left|\varphi\left(w_{n}\right)\right| \geq \exp \left(n^{2}\left|w_{n}\right|\right), \quad n \in \mathbb{N} .
$$

As before, we may assume that $\left\{w_{n}\right\}$ satisfies the assumptions of Lemma 7, after adding the point $w_{0}=0$ to the sequence. We choose $r_{n}:=1 / n$, and let $f$ be a univalent map of $\mathbb{D}$ onto the corresponding domain $\Omega$, and $z_{n}:=f^{-1}\left(w_{n}\right)$, with $z_{0}=0$. Thus $f \in \mathcal{B}_{0}$ and, as before,

$$
\begin{aligned}
\|\varphi \circ f\|_{A^{q}}^{q} & \geq\left(1-\left|z_{n}\right|\right)^{2}\left|\varphi\left(f\left(z_{n}\right)\right)\right|^{q} \\
& \geq\left(1-\left|z_{n}\right|\right)^{2} \exp \left(q n^{2}\left|f\left(z_{n}\right)\right|\right) \\
& \geq\left(1-\left|z_{n}\right|\right)^{2-c q n} \\
& \geq\left(1-\left|z_{n}\right|\right)^{-1}
\end{aligned}
$$

whenever $n>3 / c q$, thus giving the desired contradiction. 
It remains to prove that all of these operators are continuous. Since $\|\cdot\|_{A^{q}}$ is an increasing function of $q$, we may assume that $q \geq 1$. We fix $\varphi \in E(p /(p-1))$. It follows that also $\varphi^{\prime} \in E(p /(p-1))$; see [T, 8.51]. Thus we can choose constants $C, \alpha$ such that $\left|\varphi^{\prime}(z)\right| \leq C \exp \left(\alpha|z|^{p /(p-1)}\right)$ for all $z \in \mathbb{C}$.

Let us fix $f \in B^{p}$. By calculus, there exists a convex linear combination $F(z)$ of $f(z)$ and $g(z)$ such that

$$
|\varphi(f(z))-\varphi(g(z))| \leq|f(z)-g(z)| \cdot\left|\varphi^{\prime}(F(z))\right| .
$$

By Cauchy-Schwarz we deduce that

$$
\int_{\mathbb{D}}|\varphi \circ f-\varphi \circ g|^{q} d A \leq\left(\int_{\mathbb{D}}|f-g|^{2 q} d A\right)^{1 / 2}\left(\int_{\mathbb{D}}\left|\varphi^{\prime} \circ F\right|^{2 q} d A\right)^{1 / 2} .
$$

By (11), we see that the first factor on the right is at most $C\|f-g\|_{B^{p}}^{q}$, so continuity follows if we show that the second factor is bounded when $\|f-g\|_{B^{p}}$ is sufficiently small.

Since

$$
|F|^{t} \leq(|f|+|g|)^{t} \leq 2^{t-1}\left(|f|^{t}+|g|^{t}\right), \quad t \geq 1,
$$

it follows from the growth estimate on $\varphi^{\prime}$ and Cauchy-Schwarz that

$$
\begin{aligned}
& \int_{\mathbb{D}}\left|\varphi^{\prime} \circ F\right|^{2 q} \leq C^{2 q} \int_{\mathbb{D}} \exp \left(2^{p /(p-1)} q \alpha|f(z)|^{p /(p-1)}\right) \cdot \exp \left(2^{p /(p-1)} q \alpha|g(z)|^{p /(p-1)}\right) d A \\
\leq & C^{2 q}\left(\int_{\mathbb{D}} \exp \left(2^{(2 p-1) /(p-1)} q \alpha|f|^{p /(p-1)}\right)\right)^{1 / 2} \cdot\left(\int_{\mathbb{D}} \exp \left(2^{(2 p-1) /(p-1)} q \alpha|g|^{p /(p-1)}\right)\right)^{1 / 2} .
\end{aligned}
$$

The boundedness of both of these factors (by a quantity independent of $g$ ) follows from Theorem 3(b) as long as

$$
\|f-g\|_{B^{p}}<r<\left(\frac{s_{p}}{2^{(2 p-1) /(p-1)} q \alpha}\right)^{(p-1) / p} .
$$

Replacing Theorem 3(b) by Theorem 6(b), the argument for $\mathcal{B}_{0}$ is very similar to that for $B^{p}$, so we omit it.

REMARK 8. Since $H^{q} \subset A^{q}$, it immediately follows from the above theorem that $\varphi \in$ $E(p /(p-1))$ is a necessary condition for $S_{\varphi}\left(B^{p}\right) \subset H^{q}$ whenever $1<p<\infty$ and $0<q<\infty$. However we do not know if it is sufficient. An exception is the case $p=2$, since we can then use Theorem 5 in place of Theorem 3; the key difference is that we integrate over $\mathbb{T}$ rather than $\mathbb{D}$. These superposition operators can be shown to be continuous in a similar fashion.

REMARK 9. It is clear from the above proof of continuity, that superposition operators $S_{\varphi}: X \rightarrow A^{q}$, where $X=B^{p}$ or $X=\mathcal{B}_{0}$, actually satisfy the following local Lipschitz condition:

$$
\left\|S_{\varphi}(g)-S_{\varphi}(f)\right\|_{A^{q}} \leq C_{f}\|g-f\|_{X}
$$


provided that $\|g-f\|_{X}<r$, where $r>0$ is independent of $f, g$. Note that the local Lipschitz constant $C_{f}$ depends essentially on $f$. See $[\mathrm{CG}]$ for analogous results for $A^{p}$.

4.2. Operators bounded from $B^{p}$ to $A^{q}$. Finally, we prove Theorem 2, thereby characterizing the bounded superposition operators from $B^{p}$ or $\mathcal{B}_{0}$ to $A^{q}$. Since the answer is independent of $q$ and $A^{r}$ embeds compactly in $A^{q}$ whenever $0<q<r$, such bounded operators are automatically Montel compact (i.e., they send bounded sets to relatively compact sets). We note that the bounded operators from $\mathcal{B}$ to $A^{q}$ were characterized in $[\mathrm{AMV}]$ and are easily seen to be compact because of the compactness of injections from smaller into larger Bergman spaces.

Proof of Theorem 2. To prove sufficiency, assume that $\varphi \in E_{0}(p /(p-1))$. We need to show that for all $C>0$, there exists a constant $K$ such that

$$
\sup \left\{\int_{\mathbb{D}}|\varphi \circ f|^{q} d A:\|f\|_{B^{p}} \leq C\right\} \leq K .
$$

If $0<\alpha \leq c=c(p)$, then Theorem 3(a) implies that

$$
\sup \left\{\int_{\mathbb{D}} \exp \left(\alpha|g|^{p /(p-1)}\right) d A:\|g\|_{B^{p}} \leq 1\right\} \leq 2 .
$$

Let us suppose that $\|f\|_{B^{p}} \leq C$, and choose $\varepsilon=c / C^{p /(p-1)} q$, where $c$ is as in (19). Since $\varphi \in E_{0}(p /(p-1))$, there exists a positive number $R_{0}$ such that $|\varphi(w)| \leq \exp \left(\varepsilon|w|^{p /(p-1)}\right)$ whenever $|w|>R_{0}$. Applying (19), we see that

$$
\begin{aligned}
\int_{\mathbb{D}}|\varphi \circ f|^{q} d A & =\int_{\left\{z:|f(z)| \leq R_{0}\right\}}|\varphi \circ f|^{q} d A+\int_{\left\{z:|f(z)|>R_{0}\right\}}|\varphi \circ f|^{q} d A \\
& \leq M\left(R_{0}, \varphi\right)^{q}+\int_{\mathbb{D}} \exp \left(q \varepsilon|f(z)|^{p /(p-1)}\right) d A(z) \\
& \leq M\left(R_{0}, \varphi\right)^{q}+2 .
\end{aligned}
$$

Since $M\left(R_{0}, \varphi\right)$ depends only upon $\varphi, p$, and $C$, the boundedness of $S_{\varphi}$ is proved.

The $\mathcal{B}_{0}$ case follows from [AMV, Theorem 3] which says that the bounded superposition operators from $\mathcal{B}_{0}$ to $A^{q}$ are precisely those $S_{\varphi}$ for which $\varphi \in E_{0}(1)$.

We now prove necessity. Assume, on the contrary, that $S_{\varphi}$ maps $B^{p}$ boundedly into $A^{q}$ but $\varphi \notin E_{0}(p /(p-1))$. Thus there exists a number $\sigma>0$ and a sequence $\left\{w_{n}\right\}$ in the plane such that $1<\left|w_{n}\right| \rightarrow \infty$ and

$$
\left|\varphi\left(w_{n}\right)\right| \geq \exp \left(\sigma\left|w_{n}\right|^{p /(p-1)}\right), \quad n \in \mathbb{N} .
$$

Now choose $C$ large enough so that $q \sigma C^{p /(p-1)}>2$. Since $S_{\varphi}$ is bounded, there exists a constant $K$ with the property that $\|\varphi \circ f\|_{A^{q}}^{q} \leq K$ whenever $\|f\|_{B^{p}} \leq C$. Since $\left|w_{n}\right| \rightarrow \infty$, for each $n$ we can find a (unique) $r_{n}$ in the interval $[0,1)$ such that

$$
\left|w_{n}\right|=\left(\log \frac{1}{1-r_{n}^{2}}\right)^{1-1 / p}
$$


Recalling the definition of $f_{r, p}$ and $c_{r, p}$ given in (8), we define $c_{n}:=c_{r_{n}, p}$ and

$$
f_{n}(z):=C e^{i \arg w_{n}} \frac{\log \frac{1}{1-r_{n} z}}{\left(\log \frac{1}{1-r_{n}^{2}}\right)^{1 / p}}=C c_{n}^{-1} e^{i \arg w_{n}} f_{r_{n}, p} .
$$

Then $f_{n}$ has the properties

$$
f_{n}\left(r_{n}\right)=C w_{n}, \quad\left|f_{n}\left(r_{n}\right)\right|=C\left(\log \frac{1}{1-r_{n}^{2}}\right)^{1-1 / p} .
$$

Moreover, each $f_{n} \in B^{p}$ and has $B^{p}$ norm $C / c_{n}$, which is uniformly bounded.

Using the boundedness assumptions on $S_{\varphi}$, together with (5), (20), and (21), we have

$$
\begin{aligned}
K \geq\left\|\varphi \circ f_{n}\right\|_{A^{q}}^{q} & \geq\left(1-r_{n}\right)^{2}\left|\varphi\left(f_{n}\left(r_{n}\right)\right)\right|^{q} \\
& \geq\left(1-r_{n}\right)^{2} \exp \left(q \sigma\left|f_{n}\left(r_{n}\right)\right|^{p /(p-1)}\right) \\
& \geq\left(1-r_{n}\right)^{2} \exp \left(q \sigma C^{p /(p-1)} \log \frac{1}{1-r_{n}^{2}}\right) \\
& \gtrsim\left(1-r_{n}\right)^{2-q \sigma C^{p /(p-1)}},
\end{aligned}
$$

which is impossible in view of our choice of $C$.

The $\mathcal{B}_{0}$ case is similar. If $S_{\varphi}$ maps $\mathcal{B}_{0}$ boundedly into $A^{q}$ but $\varphi \notin E_{0}(1)$, then there exists $\sigma>0$ and $\left\{w_{n}\right\}$ such that $1<\left|w_{n}\right| \rightarrow \infty$ and

$$
\left|\varphi\left(w_{n}\right)\right| \geq \exp \left(\sigma\left|w_{n}\right|\right), \quad n \in \mathbb{N} .
$$

Now choose $C>2 / q \sigma$. Since $S_{\varphi}$ is bounded, there exists a constant $K$ with the property that $\|\varphi \circ f\|_{A^{q}}^{q} \leq K$ whenever $\|f\|_{B^{p}} \leq C$. For each $n$ we can find a (unique) $r_{n} \in[0,1$ ) such that

$$
\left|w_{n}\right|=\frac{1}{2} \log \frac{1+r_{n}^{2}}{1-r_{n}^{2}}
$$

Let

$$
f_{n}(z):=\frac{C e^{i \arg w_{n}}}{2} \log \frac{1+r_{n} z}{1-r_{n} z} .
$$

Then $f_{n}$ has the properties

$$
f_{n}\left(r_{n}\right)=C w_{n}, \quad\left|f_{n}\left(r_{n}\right)\right|=C \log \frac{1+r_{n}^{2}}{1-r_{n}^{2}}
$$

Moreover, each $f_{n} \in \mathcal{B}_{0}$ and $\left\|f_{n}\right\|_{\mathcal{B}_{0}} \leq C$. 
As before, we then get

$$
\begin{aligned}
K \geq\left\|\varphi \circ f_{n}\right\|_{A^{q}}^{q} & \geq\left(1-r_{n}\right)^{2}\left|\varphi\left(f_{n}\left(r_{n}\right)\right)\right|^{q} \\
& \geq\left(1-r_{n}\right)^{2} \exp \left(q \sigma\left|f_{n}\left(r_{n}\right)\right|\right) \\
& \geq\left(1-r_{n}\right)^{2} \exp \left(q \sigma C \log \frac{1+r_{n}^{2}}{1-r_{n}^{2}}\right) \\
& \gtrsim\left(1-r_{n}\right)^{2-q \sigma C},
\end{aligned}
$$

which is impossible in view of our choice of $C$.

As mentioned earlier, Montel compactness follows from compactness of the embedding of $A^{r}$ in $A^{q}$.

\section{REFERENCES}

[AMV] V. Álvarez, M. A. Márquez, and D. Vukotić, Superposition operators between the Bloch space and Bergman spaces, Ark. Mat. 42 (2004), No. 2, 205-216.

[ACP] J.M. Anderson, J. Clunie and Ch. Pommerenke, On Bloch functions and normal functions, J. Reine Angew. Math. 270 (1974), 12-37.

[AZ] J. Appell and P. P. Zabrejko, Nonlinear Superposition Operators, Cambridge University Press, Cambridge, 1990.

[AFP] J. Arazy, S. D. Fisher, and J. Peetre, Möbius invariant function spaces, J. Reine Angew. Math. 363 (1985), 110-145.

[Ax] S.J. Axler, Zero multipliers of Bergman spaces, Canad. Math. Bull. 28 (1985), 237-242.

[Be] A. Beurling, Études sur un problème de majoration, Thèse pour le doctorat, Almquist \& Wieksell, Upsalla, 1933.

[Boa] R. P. Boas, Jr., Entire functions, Academic Press Inc., New York, 1954.

[Boe] B. Böe, Interpolating sequences for Besov spaces, J. Funct. Anal. 192 (2002), 319-341.

[BFV] S. M. Buckley, J. L. Fernández, and D. Vukotić, Superposition operators on Dirichlet type spaces, Report Univ. Jyväskylä 83 (2001), 41-61. (Papers on Analysis: dedicated to Olli Martio on the occasion of his 60th Birthday, editors: J. Heinonen, T. Kilpeläinen, and P. Koskela.) Available from http://www.math.jyu.fi/research/report83.html.

[BO] S. M. Buckley and J. O'Shea, Weighted Trudinger-type inequalities, Indiana Univ. Math. J. 48 (1999), 85-114.

[BV] S. M. Buckley and D. Vukotić, Superposition operators and the order and type of entire functions. In: Recent advances in operator-related function theory, 51-57, Contemp. Math. 393, Amer. Math. Soc., Providence, RI, 2006.

[Ca] G. Cámera, Nonlinear superposition on spaces of analytic functions, in: Harmonic analysis and operator theory (Caracas, 1994), 103-116, Contemp. Math. 189, Amer. Math. Soc., Providence, RI, 1995.

[CG] G. Cámera and J. Giménez, Nonlinear superposition operators acting on Bergman spaces, Compositio Math. 93 (1994), 23-35.

[Ch] S.-Y. A. Chang, The Moser-Trudinger inequality and applications to some problems in conformal geometry. In Nonlinear Partial Differential Equations in Differential Geometry (R. Hardt and M. Wolf, editors), Park City, Utah 1992, 65-125, IAS/Park City Math. Ser., 2, American Mathematical Society, Providence, Rhode Island, 1996.

$[\mathrm{CM}]$ S.-Y. A. Chang and D. E. Marshall, On a sharp inequality concerning the Dirichlet integral, Amer. J. Math. 107 (1985), 1015-1033. 
[DGV] J. J. Donaire, D. Girela, and D. Vukotić, On univalent functions in some Möbius invariant spaces, J. Reine Angew. Math. 553 (2002), 43-72.

[D] P. L. Duren, Theory of $H^{p}$ Spaces, Academic Press, New York-London 1970. Reprint: Dover, Mineola, New York, 2000.

[DS] P. L. Duren and A. P. Schuster, Bergman Spaces, Math. Surveys and Monographs 100, American Mathematical Society, Providence, Rhode Island, 2004.

[HL] G. H. Hardy, J. E. Littlewood, Some properties of fractional integrals. II, Math. Z. 34 (1932), 403-439.

[HKZ] H. Hedenmalm, B. Korenblum, and K. Zhu, Theory of Bergman Spaces, Graduate Texts in Mathematics, Vol. 199, Springer, New York, 2000.

[HW] F. Holland and D. Walsh, Growth estimates for functions in the Besov spaces $A_{p}$, Proc. Roy. Irish Acad. Sect. A $\mathbf{8 8}$ (1988), 1-18.

[P] Ch. Pommerenke, Boundary Behaviour of Conformal Maps, Springer-Verlag, Berlin, 1992.

[T] E. C. Titchmarsh, The Theory of Functions, 2nd edition, Oxford University Press, Oxford, 1988.

[W] D. Walsh, A property of univalent functions in $A_{p}$, Glasgow Math. J., 42 (2000), 121-124.

[X] C. Xiong, Superposition operators between $Q_{p}$ spaces and Bloch-type spaces, Complex Var. Theory Appl. 50 (2005), no. 12, 935-938.

[Z] K. Zhu, Analytic Besov Spaces, J. Math. Anal. Appl. 157 (1991), 318-336.

Department of Mathematics, National University of Ireland, Maynooth, Co. KilDARE, IRELAND

E-mail address: sbuckley@maths.nuim.ie

Departamento de Matemáticas, C-XV, Universidad Autónoma de Madrid, 28049 Madrid, SPAIN

E-mail address: dragan.vukotic@uam.es 